\title{
EL TIEMPO ENTRE TRAYECTORIAS Y TRANSFORMACIONES
}

\section{THE TIME BETWEEN TRAJECTORIES AND TRANSFORMATIONS}

\author{
Jorge Eliécer Molina Zapata
}

Fecha de recibido: Febrero 3 de 2010

Correspondencia: Programa de Filosofía, Universidad del Quindío Av. Bolivar calle 12 norte Armenia Quindío. Correo electrónico: jemolina@uniquindio.edu.co

\section{RESUMEN}

El carácter de la ciencia o del pensamiento contemporáneo fluye en función del "papel constructivo del tiempo» en procesos irreversibles, los mismos que sustentan lo que ha sido la evolución de la vida y la biodiversidad en el planeta. En tal sentido, este concepto de tiempo acontece como un «cambio revolucionario» para nuestra cultura en tanto nos permite percibir un mundo donde lo humano no es extraño a la naturaleza tal como ocurría con el dominio explicativo de la ciencia clásica. De ahí que, aun considerando la innegable utilidad y las comodidades que el desarrollo de la ciencia moderna nos ha concedido, actualmente no es sustentable un pensamiento simplista dada la naturaleza altamente compleja de los problemas que nos sobrecogen. En efecto, las condiciones de vida del entorno social y ecológico de nuestra época, en todos sus niveles y planos, demandan transiciones en los modos como habitamos el mundo, pues en ello se juega la viabilidad de la especie humana en el devenir de la vida en general. Es sorprendente percibir cómo ha cambiado la concepción de la ciencia en tan corto tiempo al punto que es posible afirmar un nuevo pensamiento científico en torno a la diversidad e inestabilidad, la aurora de un dominio explicativo que se muestra jovial y fecundo.

Palabras clave: Tiempo, causalidad, determinismo, cronología, trayectoria, devenir, trasformación, termodinámica, estructura disipativa, bifurcación, diferencia.

\section{ABSTRACT}

The nature of science and contemporary thought flows on the basis of the "constructive role of time" in irreversible processes, the same that supports what has been the evolution of life and biodiversity on the planet. In that sense, this concept of time occurs as a "revolutionary change" for our culture, meanwhile we can perceive a world where the human things are not foreign to nature as happened with the explanatory domain of classical science. Hence, even considering the undeniable utility and amenities that the development of modern science has given us, actually is not sustainable a simplistic thinking given the highly complex nature of the problems that overwhelm us. In fact, the living conditions of society and the environment of our time at all levels, demand transitions in the ways we inhabit the world, because in this it is played the viability of the human species in the evolution of life. It is surprising how it has changed the conception of science in such a short time to the point that it is possible to affirm a new scientific thinking about the diversity and instability, the dawning of an explanatory domain looks jovial and fruitful

Keywords: Time, causality, determinism, chronology, trajectory, becoming, transformation, thermodynamics, dissipative structures, bifurcation, difference.

\section{INTRODUCCIÓN.}

Difícilmente encontremos en la evolución del pensamiento científico un concepto que exprese con mayor vivacidad su cambio como lo ha hecho el concepto del tiempo. Cada momento decisivo en la historia de las ciencias ha tenido que vérselas con este concepto y como resultado de tal encuentro surge un modo particular del interactuar humano occidental con y en el mundo. Es decir, la concepción del tiempo ha sido condición inicial para las interacciones de lo humano entre sí y con la naturaleza en cualquier época de nuestra cultura; obviamente incluyendo lo diferencialmente contemporáneo que se expresa en problemas y desafíos revolucionarios (en el sentido khuniano del término) del pensar en todas sus interdependientes extensiones.

Así, por ejemplo, se comprende que para la ciencia clásica, o de mecánica de partículas, se confunda el reloj con el tiempo, pues desde la antigüedad (siguiendo el camino ${ }^{1}$ transitado que dio carácter a la cultura occidental, babilonios y egipcios, y su herencia a los presocráticos milesios, eléatas y jónicos) el tiempo se comprendió como duración: de las estaciones del año, de la luz en el día y de la oscuridad en la noche, de las mareas, entre otros fenómenos. No obstante los pitagóricos desarrollaron otro alcance a esta idea del tiempo al relacionarlo con la periodicidad; pues vieron en esta noción el carácter armónico y rítmico del cosmos (kosmos), análogo al de la música; así como la expresión de la estructura matemática (mathemata) de éste en la legalidad de la

\footnotetext{
Docente del Programa de Filosofía e investigador del grupo interdisciplinario de investigación "Bioética, ciencias y cultura de lo viviente" de la Universidad del Quindío.

${ }^{1}$ Sólo actualmente dimensionamos los alcances del camino propuesto por Heráclito, Demócrito, Epicuro y Lucrecio, rechazado más de veinte cinco siglos para darle continuidad, sin más, al estatismo de Parménides.
} 
naturaleza (physis)

Entonces el problema del tiempo es resuelto como una serie repetible de ciclos a la manera del movimiento de cuerpos celestes, de la sucesión de generaciones humanas o, dicho de otro modo, como parámetro del movimiento cíclico: armonía cósmica entre el tiempo monocorde y el espacio abstracto. Este idealismo matemático tuvo ulteriores connotaciones en el ser permanente de Parménides, el eidos de Platón y la sustancia de Aristóteles, entre otros pensadores y propuestas de significativa influencia conceptual en la consolidación de la estructura de la ciencia moderna. Así, pues, el árbol del conocimiento de Descartes echó sus raíces en tierra labrada sin tregua con el mismo arado desde la antigüedad y creció en búsqueda de la luz de la razón en función de la certeza y el control. No podía esperarse otro resultado.

Como vemos, históricamente nuestra cultura ha pensado el tiempo como complemento del espacio, es decir, como extensión que a su vez se sistematiza en instantes que transcurren $y$, al igual que el espacio, el tiempo se hace susceptible de medición en términos de magnitudes numéricas. En efecto, para el pensamiento clásico, tiempo y espacio son descritos fundamentalmente en el lenguaje del cálculo, en particular por ecuaciones lineales con las cuales es posible la regresión de un proceso: entre tiempo pasado y futuro no aparece, cualitativamente, diferencia alguna. Además, tales ecuaciones, asumidas entonces como universales y necesarias, son el sustento de las leyes científicas de la dinámica determinista. De este modo, poco a poco, se estableció la imagen del mundo sólo como un objeto de estudio susceptible de ser controlado una vez se conozcan las claves del encadenamiento causal de su funcionamiento: autómata, desencantado y trivial, dicen algunos. En definitiva, el mundo se concibió dinámicamente mecánico y, en coherencia, temporalmente simétrico.

Ahora bien, situación asombrosamente diferente encontramos en la ciencia contemporánea, cuyo mayor interés estriba en el estudio de sistemas abiertos alejados del equilibrio, aquellos cuyo comportamiento expresan «fluctuaciones, inestabilidad y autoorganización» fruto de las interacciones no-lineales de sus componentes, entre sí y con su entorno. Igualmente, son sistemas que evolucionan con el tiempo, esto es, de manera irreversible y en aumento de su complejidad. Por lo anterior, no permiten ser descritos sólo por números o cantidades porque sus cambios son estructurales y éstos, a su vez, generan nuevas pauta en sus comportamientos. En tal sentido, se vienen desarrollando las matemáticas cualitativas, a las cuales nos referiremos más adelante, para comprender cambios morfológicos, discontinuos o catastróficos en dichos sistemas. Esto, característico de sistemas de comportamiento complejo, nos habla de un tiempo como devenir, el cual incluye la novedad como posibilidad de sus componentes.

El propósito de las siguientes páginas es sustentar la tesis, reiteradamente implícita en la literatura científica actual, de que las rupturas que han venido presentándose entre la ciencia clásica y la contemporánea están principalmente originadas en el cambio de la concepción del tiempo y sus notables alcances ontológicos - epistemológicos y lógicos que se expresan vivamente, entre otras, en la creciente borrosidad de la distinción entre ciencias naturales y ciencias humanas como ciencias duras y blandas respectivamente. Quiere decir, pasar de la reversibilidad de los procesos del paradigma mecano-determinista a la irreversibilidad de sistemas que evolucionan en y con el tiempo entre el caos y las inestabilidades de sus interacciones en continuos feed-backs, esto es, en términos de Prigogine, un mundo en no-equilibrio, abierto a la innovación.

En el desarrollo de este artículo se explican algunos de los conceptos y categorías más importantes de los dominios explicativos de la ciencia clásica y de las ciencias contemporáneas en torno al concepto del tiempo en cada uno de ellos, a fin de exponer sus diferencias y sus alcances. Por tanto, en función de la tesis propuesta, se hace una indagación concerniente a la relación entre la cultura científica y la cultura humanista.

\section{LA NEGACIÓN DEL TIEMPO EN LA LINEALIDAD: LA RAZÓN DE ESPALDAS A LA VIDA.}

Partamos de una pregunta como guía de recorrido por este primer momento: ¿qué se hace necesario para sujetar la excitable voluntad humana estimulada además por el placer que producen los sentidos y, a la vez, simplificar la complejidad creciente de la naturaleza en función de un conocimiento certero que permita controlarla? Evidentemente la pregunta oculta una historia vinculada a una particular manera de hacer ciencia que poco a poco, generación tras generación, estructuró el carácter de la cultura occidental, esto es, su representación o imagen del mundo $y$, en congruencia, su modo de interactuar o comportarse en el mismo. A partir de allí condiciona sus modos de transformación o de cambio, en definitiva, su modo de pensarse, o no, a futuro.

El marco conceptual de la ciencia clásica, prolongado en la relatividad y, bajo ciertas interpretaciones ontológicas ${ }^{2}$, en la mecánica cuántica, está finamente sustentado en dos creencias, entre otras de menor relevancia que pueden ser tomadas como derivadas o componentes de aquéllas, que fueron de manera exclusiva hilo conductor del pensamiento en occidente hasta bien entrado el siglo XX y lo siguen siendo hoy para algunos que, de nuevo en términos de Khun (2004), hacen parte de alguna comunidad académica e investigativa cuyos productos encuadran en el "desarrollo normal o acumulativo" de la ciencia y (o) la filosofía. Puntualmente, estamos hablando de la creencia obsesiva en la causalidad y en el pensamiento analítico como productoras de verdades universal y necesariamente válidas.

Se trata de los fundamentos con los cuales se abordó el problema de la realidad, la manera como a ésta se la concibió por un largo período de nuestra historia y con los que se exacerbó cierto carácter de nuestra cultura. Dicho carácter se destaca por la racionalización o matematización del mundo por medio de modelos (teorías, técnicas, métodos o

\footnotetext{
${ }^{2}$ Véase, por ejemplo, Penrose, R. (2007). El camino a la realidad. Una guía completa de las leyes del universo. México: Debate.
} 
metodologías) que, reduciendo al máximo el comportamiento complejo de la naturaleza y, por extensión, de los colectivos humanos, enuncian una explicación simplificadora de éstos. La particularidad de este dominio explicativo es, en lo esencial, el cálculo de trayectorias a través de ecuaciones expresables exclusivamente en entidades numéricas. Hay que destacar, entonces, que el largo camino de y hacia la ciencia clásica exigió la sustitución paulatina del concepto por la fórmula; exigencia que se extiende a todo el pensamiento moderno.

Un primer modo en la caracterización de esta simplicidad se manifiesta con la relación de causalidad, la cual denota dos componentes básicos: linealidad y simetría. La causalidad dice, en términos generales, que "todo ente contingente tiene una causa" o, dicho de otra manera, que "cada efecto tiene una causa." Además, que entre causa y efecto hay a) relación lineal, en el sentido de una dependencia funcional (léase en términos aristotélicos como eficiente) invariable, donde grandes efectos ( $p$. ej. movimientos o desplazamientos) son consecuencia de grandes causas (p. ej. fuerzas o vectores) y donde no podemos esperar cambios considerables suscitados por pequeñas influencias: lo grande produce lo grande y lo pequeño a su semejante. Dicho de otro modo, los movimientos son proporcionales a las fuerzas involucradas. De otra parte, $\boldsymbol{b}$ ) tenemos simetría en tanto podemos hacer la regresión de efecto a causa hasta llegar a la causa primera donde la modernidad en sus inicios ubicó un ser transcendental (a la manera del Dios judeo cristiano) como legislador racional del universo y, por tanto, pináculo de la racionalidad de la dinámica causal, en la forma del principio ontológico y lógico de razón suficiente (Leibniz, 1982).

El pensamiento analítico constituye el segundo modo en la caracterización de esta simplicidad. El análisis, como método que consiste en aislar algo para estudiarlo y comprenderlo, se instaura como vía regia a partir de Descartes, plantea que la complejidad del mundo puede comprenderse plenamente a partir de las propiedades de sus partes aisladas. De este modo, busca describir un universo transparente, inequívoco y abierto a la evidencia por medio de las «ideas claras y distintas»: el pensamiento geométrico como el método (camino) que rige el uso correcto del «buen sentido». El espíritu del pensamiento analítico es, pues, la desintegración del mundo o del todo (considerado una sumatoria de partes) hasta llegar a sus elementos más simples, los «ladrillos últimos» de los está construida la realidad.

La concepción de un mundo mecánico y temporalmente reversible (siempre idéntico, con procesos de enlace o sucesión temporal, de sistemas aislados, de certitud), permitió la postulación del determinismo más pretensioso, alcanzable a través de una inteligencia infinita que pudiera conocer plenamente el estado del universo sin distinción entre pasado y futuro:

Así pues, hemos de considerar el estado actual del universo como el efecto de su estado anterior y como la causa del que ha de seguirle. Una inteligencia que en un momento determinado conociera todas las fuerzas que animan a la naturaleza, así como la situación respectiva de los seres que la componen, si además fuera lo suficientemente amplia como para someter a análisis tales datos, podría abarcar en una sola fórmula los movimientos de los cuerpos más grandes del universo y los del átomo más ligero; nada le resultaría incierto y tanto el futuro como el pasado estarían presentes ente sus ojos (Laplace, 1995, p. 25).

Respecto a la causalidad, hay que mencionar, además, que el supuesto vínculo ontológico, entre un fenómeno actual y un fenómeno futuro, que configura la relación causalidad necesidad, está en estrecha afinidad con la epistemología causal que también condujo al problema de la inducción, a saber, ¿cómo justificar lógicamente la transformación de unas observaciones particulares en leyes universales y necesarias? o ¿cómo justificar racionalmente que observada una supuesta causa podamos esperar invariablemente, y para siempre, un determinado efecto?

Pues bien, históricamente fue Hume (1984), en una estética expresión de sensatez, quien primero da el martillazo para el debilitamiento de ese supuesto nexo ontológico entre causa y efecto: presunta conexión necesaria entre fenómenos que servía no sólo como justificación a la idea de substancia, sino también como principio de validez universal ${ }^{3}$ al conocimiento científico elevándolo a la condición de necesario (es decir, aquello que no puede ser de otra manera). Para este filósofo de Edimburgo la relación causal no es más que fruto de la costumbre, pues sólo por la repetición de casos en que de manera sucesiva y contigua se presenten los mismos fenómenos, llegamos a creer que un fenómeno conlleva necesariamente a otro. Así tenemos que, para Hume, ontológica y epistemológicamente la relación causa y efecto es sólo fruto de la costumbre fundada en el hábito. Esta conclusión deja la encumbrada razón y su necesidad de conexión causal atareada en el escepticismo con respecto a así misma y a los sentidos.

De ahí que, por ejemplo, Kant (1998, pp. 102-255) desarrolló su propuesta del mundo nouménico: de lo espaciotemporalmente indeterminable, como la libertad, para superar ese problema en sentido dogmático. Ubica entonces la causalidad y el determinismo como propios del mundo fenoménico siguiendo la propuesta ${ }^{4}$ de la síntesis newtoniana. A la vez que ésta se asumió como modelo de las ciencias que quisieran adquirir dicho estatus: aquel que reduce la complejidad del mundo a la simplicidad de la mecánica de sistemas conservativos y reversibles y, paralelamente, circunscribe toda afección humana al modelo teoremático.

En efecto, estamos hablando de sistemas conservativos, aquellos que, cuando no actúan influencias exteriores, permanecen constantes a lo largo del tiempo (hacia adelante

\footnotetext{
Conviene precisar que ambos componentes de esta conexión tienen sus orígenes en Aristóteles: Libro VII y Libro I de la Metafísica, respectivamente Como es sabido Kant fundamenta filosóficamente la ciencia newtoniana como saber que se independiza de la filosofía o la metafísica, las ciencias se dedicarán al estudio de los fenómenos espacio-temporales determinables al definir su objeto de conocimiento, su pauta metodológica y su propio lenguaje científico.
} 
t o hacia atrás $\mathrm{t}^{\prime}=-\mathrm{t}$ ) las siguientes magnitudes: la energía total, el momento total y el momento angular total (o momento del movimiento angular). Algunos de sus ejemplares más arquetípicos los tenemos en el péndulo, el oscilador armónico, la propagación de la luz en el vacío (en el campo del electromagnetismo de Maxwell), el problema de los dos cuerpos, entre otros, en síntesis, en todos los desarrollos propios y ulteriores de la teoría newtoniana.

\section{El tiempo de las leyes deterministas, concepto métrico: cronología.}

Como se ha indicado, el desarrollo del dominio explicativo clásico se expresa en función del perfeccionamiento de modelos descriptivos pragmáticamente eficaces, esto es, aquellos que cuantitativamente permita la localización espacio-temporal de los fenómenos que describe. La predicción certera es su mayor propósito, de modo que, para este dominio científico, sólo son relevantes los fenómenos expresables numéricamente a través de ecuaciones deterministas. Este es, grosso modo, el argumento positivista que sustenta la distinción entre ciencias exactas o naturales y ciencias sociales. Aquéllas, en particular la física, como se ha insinuado, serían el arquetipo del paradigma científico clásico (analítico y-en tanto que-cuantificador) a seguir por cualquier disciplina que aspire a acceder al rango de ciencia. Éstas, las ciencias sociales, frenéticamente buscaron igualarse, epistemológica y metodológicamente ${ }^{5}$, desechando así las posibilidades heurísticas de su singularidad.

En definitiva, descartar, desmembrar, desvincular, reducir grados de libertad como se dice en jerga especializada, son prácticas cotidianas del pensamiento clásico. Condición necesaria para eliminar en el sistema estudiado cualquier intercambio 'extraño' de información con su entorno y así determinar (el verbo más usado en proyectos de investigación) la dinámica y sus resultados ¿Qué se investiga entonces? Cambios de cantidades, datos numéricos y, al incorporar mínimos "errores", probabilidades. En la mecánica de sólidos los componentes de un sistema (partículas o individuos, da igual) no cambian cualitativamente en el tiempo, éste es externo, sólo cronología, no existe en estas ciencias el vector tiempo. Partículas o individuos se conservan idénticos entre pasado y futuro.

Adviértase, en conclusión, que el determinismo sólo es posible para un observador externo: ciencia que estudia la naturaleza confinándola en la experimentación y el laboratorio, es decir, dominándola y sometiéndola a criterios preestablecidos; excluyendo del saber, y de las posibilidades de comprensión, lo incontrolable. Michel Serres, en su texto sobre Lucrecio, describe el proceder de este pensamiento de la siguiente manera:

De modo que nadie lee los Meteoros, ni los de Lucrecio, ni los de Descartes ni los de ningún otro. ¿Por qué este rechazo? Porque los filósofos, los historiadores, los amos de la ciencia, sólo se preocupan de la vieja noción de ley. De la determinación exacta, la sobredeterminación rigurosa y el dios de Laplace. Del control absoluto y, por tanto, de la dominación inconclusa y sin márgenes, del poder y del orden. El tiempo que hace o que hará excede por completo de su contabilidad, en la que no se tiene en cuenta por ser el lugar del desorden y lo imprevisible, de lo fortuito local y lo amorfo. Es el tiempo de otro tiempo. El tiempo de las nubes que no es preciso tener en mente, no sea que se llene de nubes la cabeza. Y, sin embargo, ¿de qué sirve conocer casi al segundo el momento de un eclipse si un nublado nos impide verlo? ¿Para qué sirven todas las herramientas si la nieve y el lodo impiden su uso? Esta es la razón de que la física se haga de puertas adentro. El laboratorio y todo el sistema cerrado la protegen contra las turbulencias. La ciencia se encierra en el interior. Desde sus comienzos, va de los Meteoros al féretro, y ya no saldrá de esta clausura que excluye el azar y lo incontrolable o, como diríamos hoy, la hipercomplejidad (Serres, 1994, p. 90).

En este contexto determinista es ampliamente conocido que Einstein, quien fuera uno de los creadores de la mecánica cuántica, no pudo estar de acuerdo con sus implicaciones de indeterminación. Tal era su preferencia y convicción por una concepción determinista del mundo que en tal sentido expreso: iDios no juega a los dados! Y, como nos recuerda Prigogine, "llegó incluso a acuñar una célebre negación del tiempo, pronunciada con ocasión de la muerte de su amigo Michele Besso: «Michele se me ha adelantado en abandonar este extraño mundo. No tiene importancia. Para nosotros, físicos convenidos, la distinción entre pasado y futuro es una ilusión, aunque tenaz»" (Prigogine 1996, p. 156). De este modo la física se negó a aceptarse en la dinámica del mundo de la vida: vinculada a procesos irreversibles y a la complejidad creciente de la evolución biológica.

\section{EL TIEMPO COMO DIMENSIONES COMPONENTES $Y$ TRANSFORMADORES DE LA NATURALEZA.}

Coincidiendo en parte con Einstein, tanto Bergson como Heidegger negaron la posibilidad del tiempo en las ciencias, en la naturaleza que no fuese humana. La realidad del tiempo como devenir es propio de lo humano; sólo de su aspecto psíquico que es donde tiene lugar sus especificidades múltiples de duración, cualidad y libertad (de creación y (o)

\footnotetext{
5 "La más científica de las ciencias sociales", la economía, específicamente en su expresión ortodoxa, de corte neoclásico, es la primera de éstas que logra tal reconocimiento por la comunidad científica del paradigma moderno del saber; precisamente porque se sustenta en la normalización o estandarización de ciertos principios (de causalidad, por ejemplo), definiciones (individuo, racionalidad, decisión racional, juego, etc.) y el uso de conceptos métricos (función de utilidad, estrategia pura o mixta, equilibrio, etc.), logrando así presentarse y trabajar como un sistema matemático deductivo. La teoría de juegos clásica aportó notoriamente para tal fin.
} 
innovación); pensada así por evidencia intuitiva, diría el filósofo francés:

La sucesión existe, tengo conciencia de ella, es un hecho. Cuando un proceso físico se realiza bajo mis ojos, no depende de mi percepción ni de mi inclinación acelerarlo o demorarlo. Lo que al físico le importa es el número de unidades de duración que el proceso necesita: no hay que inquietarse por las unidades mismas y por eso los sucesivos estados del mundo podrían ser desplegados de un solo golpe en el espacio sin que su ciencia cambiase y sin que cesase de hablar del tiempo. Pero para nosotros, seres conscientes, son las unidades las que importan, porque no contamos extremidades de intervalo, sino que sentimos y vivimos los intervalos mismos. Ahora bien, tenemos conciencia de estos intervalos como de intervalos determinados. Vuelvo siempre a mi vaso de agua con azúcar: ¿por qué tengo que esperar a que el azúcar se derrita? Si la duración del fenómeno es relativa para el físico, dado que se reduce a un determinado número de unidades de tiempo, y que esas unidades mismas son lo que se quiera, esta duración para mi conciencia es un absoluto, porque coincide con un cierto grado de impaciencia que está rigurosamente determinado (Bergson, 1994, pp. 152-153).

Podemos percibir que para Bergson la física niega el tiempo en tanto no puede asumir el devenir en su dinámica conservativa. De otra parte, Heidegger a través de Ser y Tiempo hace una destrucción (Zerstörung, Destruktion) de la historia de la metafísica porque ésta ha sido el principal obstáculo a la cuestión del Ser dedicándose sólo a la explicación del ente en su conjunto u ocultando el Ser en su condición temporal. La metafísica "ha entendido el ser a partir del tiempo pero lo ha hecho, destaca Heidegger, a partir de una figura muy particular del tiempo, de la constancia o la permanencia" (Grondin, 2006, p. 315). Sin embargo el Ser en el tiempo recobrado por Heidegger, el Dasein («ser-ahí»), también se queda en el ámbito de lo humano, es decir, inadmisible en el dominio explicativo de las ciencias físicas. Para el propósito de este escrito considero innecesario detenernos en más detalles respecto al concepto de tiempo en Heidegger; baste lo anterior para lo que aquí por ahora nos interesa saber: no es la Filosofía sino las ciencias atraídas en las complejidades de algunos procesos irreversibles las que evidencian y explican el tiempo como devenir en la naturaleza.

Tiempo como degradación o la flecha del tiempo: termogonía.

Con la termodinámica en el siglo XIX aparece el tiempo en las ciencias como nuevo concepto asociado a la degradación y la irreversibilidad de los procesos físico-químicos. Su pionero fue el físico y matemático francés Sadi Carnot al proponer el calor como una forma de energía. Es notable que esta inédita expresión del tiempo surja en la misma época del pensamiento de la historia y la evolución (Lamarck, Hegel, Darwin, Marx, entre otros.). Extraño encuentro de perspectivas: de una parte, la fe en el "progreso" gradual o revolucionario y, por otro lado, el camino irreversible y sin retorno hacia lo agónico. Ejemplar bergsoniano de que la innovación estaba exclusivamente circunscrita al mundo de lo humano.

Es preciso hacer una breve explicación de la termodinámica con respecto a sus principios, los cuales, en términos generales, describen la evolución de sistemas macroscópicos de diferente naturaleza y niveles de complejidad tales como las máquinas de vapor, el crecimiento poblacional, los fenómenos climáticos, la biosfera, el universo, etc. Dichas descripciones se dan en términos de variables colectivas como la temperatura, la presión, la concentración, entre otras. Igualmente hay que tener presente que la investigación de estos principios tiene sus orígenes en estudios experimentales de motores térmicos y su aplicabilidad en la construcción de máquinas cada vez más eficientes, de ahí sus significativas aportaciones al desarrollo industrial y su coincidencia histórica con la polémica distinción entre los discursos de la ciencia «pura» y la ciencia «aplicada».

Recordemos que la descriptiva clásica se expresa en función de los sistemas conservativos, pues bien, a partir de la termodinámica trabajamos con otro tipo de sistemas que se caracterizan por el desgaste que sufren con el rozamiento: los sistemas disipativos. Con éstos acaece la irreversibilidad y la complejidad, es decir, "el fracaso del sueño laplaciano". Esto significa sostener, finalmente, que la estructura explicativa de los sistemas reversibles y deterministas no será nunca más exclusiva. Asociado al tiempo, es decir, sin regresión, un nuevo dominio de pensamiento, realmente fecundo, ha emergido. No obstante este primer momento de la termodinámica expone una concepción del mundo poco atractiva; Michel Serres la designa como «termogonía»o «biogonía».

Principio de conservación de la energía: formulado por el físico inglés James Prescott Joule en 1847, a través del cual se toma la energía, magnitud que se conserva, como un equivalente general entre calor y trabajo, es decir, entre diversas transformaciones físico-químicas en diferentes fenómenos en los cuales " "algo" se conserva cuantitativamente y cambia de forma cualitativa" (Prigogine \& Stengers, 1997, p. 145). Su enunciado diría que "si un sistema termodinámico efectúa un ciclo, es decir, una serie de transformaciones a cuyo final el sistema regresa a su estado inicial, la suma algebraica de las cantidades de calor y la suma algebraica de las cantidades de trabajo son proporcionales" (Spire, 2001, p. 14). De hecho, por ejemplo, la energía cinética perdida por un cuerpo debido al

\footnotetext{
${ }^{6}$ Ver, por ejemplo, BROWKER, Geof, El auge de la investigación industrial. En: SERRES, Michel (ed.) (1998), Historia de las Ciencias. Cátedra, Madrid.
}

Rev. Invest. Univ. Quindío (20): 78 - 88. Armenia - Colombia 
rozamiento es reciclada para calentar el aire y el cuerpo. Finalmente es así como la energía total (resultante de la composición de la energía cinética, la energía potencial y la energía térmica) se mantiene.

Para comprender mejor el segundo principio es recomendable hacer una clasificación básica de los sistemas:

- Sistemas «aislados» que no pueden intercambiar materia ni energía con el mundo externo (fig. 1).

- Sistemas «cerrados» que pueden intercambiar energía pero no materia con el mundo externo (fig. 2).

- Sistemas "abiertos» que son susceptibles de intercambiar materia, energía e información con el mundo externo (fig. 3 y 4 ).

Principio de evolución de un sistema aislado: formulado por el físico alemán Rudolph Claussius en 1865, plantea específicamente la distinción entre procesos reversibles e irreversibles. Pues, a diferencia de la igualdad de la primera ley, la segunda ley enuncia una desigualdad: en un sistema aislado, en el que se da un proceso físico-químico, una magnitud diferente, la entropía, adquiere un valor mayor o igual a cero con el transcurso tiempo el cual es irreversible. En consecuencia, el sistema olvida sus condiciones iniciales, rompe la simetría temporal y tiende inexorablemente hacia el estado de equilibrio termodinámico o máximo desorden. Esto quiere decir que el sistema se homogeniza al agotar sus diferencias.

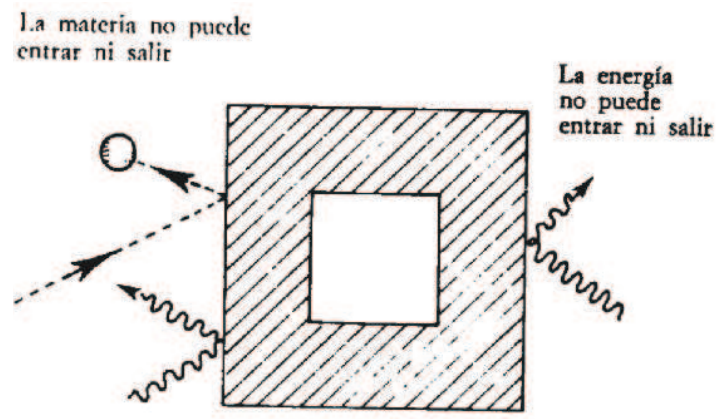

Figura 1. Sistema aislado

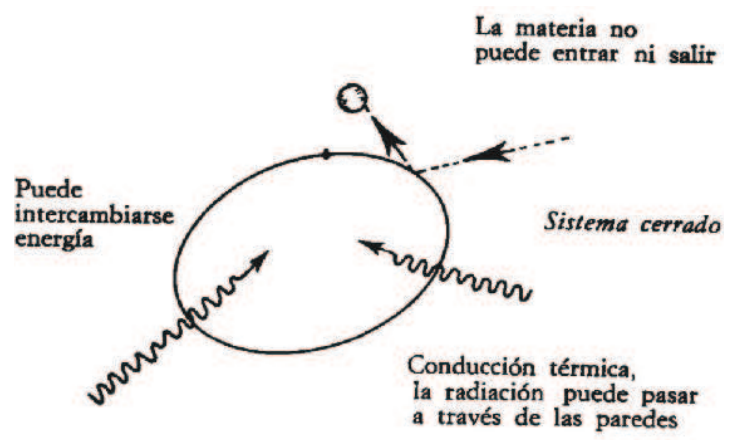

Figura 2. Sistema cerrado

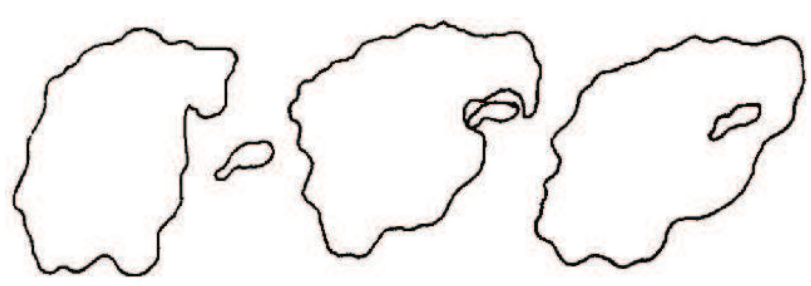

Figura 3. Una ameba fagocitando su alimento es un ejemplo de sistema abierto

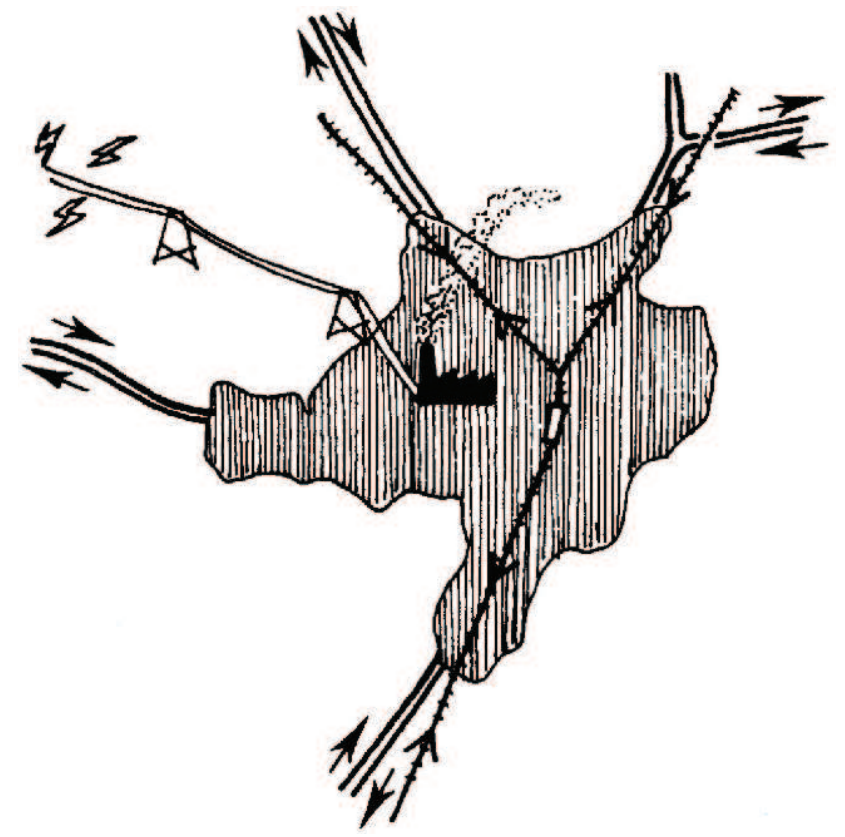

Figura 4. Una ciudad: sistema abierto

Fuente: Prigogine, 1997, pp. 221-223.

Evidentemente, «termogonía»: de una parte, la conservación de la que no es posible esperar nada nuevo; por la otra, el equilibrio, evolución en una dirección determinada tendiente a la disminución de las posibilidades en lo indiferenciado, rasgo característico de la degradación de las máquinas. Este modo de pensar de la ciencia, que privilegia la interpretación mecanicista del mundo y de la vida en términos de conservación y equilibrio, es al que a Nietzsche ${ }^{7}$, pensador de la vida, también del siglo XIX, le sobrecoge. Efectivamente, "por eso toda su crítica está basada en tres planos: contra la identidad lógica, contra la igualdad matemática, contra el equilibrio físico. Contra las tres formas de lo indiferenciado" (Deleuze, 2002, p. 67). La negación de las diferencias es, para Nietzsche, la negación de las posibilidades de la vida. Esta idea, como veremos, es robustecida en el dominio explicativo de la ciencia contemporánea.

El segundo principio de la termodinámica introduce una dirección irreversible en el tiempo ligada al envejecimiento y

\footnotetext{
Además de la obra de Deleuze sobre Nietzsche, otra buena explicación de la afección de éste respecto a la ciencia clásica se encuentra en el capítulo "Naturaleza y creatividad" (Prigogine, 1997).
} 
muerte del universo (como macro sistema aislado que expresa transformaciones físico-químicas) y, por extensión, de todos sus componentes: galaxias, estrellas, planetas y la vida en general. Ahora bien, esta tendencia a la homogenización del universo enfrenta serías dificultades, a saber, la evidente evolución y diversificación de la vida en nuestro planeta en flujos y reflujos de interacciones desde hace 3900 millones de años ${ }^{8}$ aproximadamente. En efecto, "en la física se consideraban la irreversibilidad y la disipación como una devaluación, mientras que por otro lado la evolución biológica, que es evidentemente un proceso irreversible, se relacionaba con una complejidad creciente" (Nicolis \& Prigogine 1997, p. 78). En definitiva, tal paradoja es expresión del estatus anómalo de la irreversibilidad en el "desarrollo normal o acumulativo» del dominio científico clásico.

Ciertamente hay variedad de enunciados de este principio, pero no debe asombrarnos porque, como se ha expresado, dicha variedad se debe al alcance que éste tiene en diversidad de fenómenos de distinta naturaleza. De hecho, la irreversibilidad no es exclusiva de los dominios de la física y la química, sin duda es un concepto básico en biología y sociobiología ya que todos los procesos esenciales de los seres vivos son irreversibles; obviamente, incluyendo los patrones de comportamiento colectivo de humanos y otros animales. Justamente, tal como sostiene Prigogine, el redescubrimiento del tiempo es tal vez un elemento de unidad entre ciencia, cultura y sociedad. Antiguamente la ciencia nos hablaba de leyes eternas. Hoy nos habla de historia del universo o de la materia, de ahí su aproximación evidente con las ciencias sociales y humanas.

Pues bien, la historia, como la incógnita por lo acontecido, huellas de eventos que narran la evolución de los componentes estructurales de un sistema abierto al entorno, por ejemplo, el sistema genético o inmunológico de una especie, el clima, una población animal o humana, una cultura, un concepto etc., se torna básica para comprender por qué ese sistema acontece como lo hace en la situación actual y conseguir aventurar una anticipación (prever) de cómo podría acontecer en ciertas condiciones a futuro. Es lo que hacen, por ejemplo, los meteorólogos, como lo fue Edward Lorenz, respecto a los microclimas en correlación con el sistema climático global o los geobiólogos respecto a la diversificación de especies en congruencia con la formación de los continentes y el desplazamiento de las placas tectónicas. No obstante, aunque la flecha del tiempo narra un componente básico de todo viviente como es el inexorable proceso de senectud, explica muy poco de evolución cuando se circunscribe únicamente al desarrollo temporal de sistemas aislados y (o) cerrados en los cuales se manifiesta el equilibrio como dirección fija.

Actualmente sabemos que la evolución de la vida en el planeta, en cuya base está la interacción de la biodiversidad (diversidad genética, diversidad natural: de las especies y entre individuos de las especies y diversidad cultural), como expresión del potencial creativo de la naturaleza, se hace posible en sistemas abiertos alejados del equilibrio. En otros términos, el fluir de la vida no sólo expresa deterioro, degradación y muerte (la flecha del tiempo), sino también, substancialmente, adaptabilidad, creatividad e innovación en la invención de futuros posibles (múltiples direcciones) o, como diría Deleuze, de múltiples diferencias. Lo anterior, naturalmente, es válido para las sociedades y culturas en su búsqueda de futuros, de mejores condiciones de vida colectiva en función de la heterogeneidad entre sus individuos para crear nuevas diferencias que hagan posible la actualización del flujo de la vida social ${ }^{9}$. Ahora bien, como se ha hecho en el desarrollo del texto, de nuevo no hay que tomar por dado lo que requiere explicación.

\section{El tiempo entre la entropía y la innovación: el devenir.}

Como advertimos en la introducción, desde los inicios de nuestra cultura el devenir (lo mutable) no es susceptible de conocimiento epistémico o científico. Y fue así hasta hace muy poco. Recordemos que Platón, en el Timeo, plantea: “¿Cuál es el ser eterno que no nace jamás y cuál es aquel que nace siempre y no existe nunca? El primero es aprehendido por la inteligencia y el raciocinio, pues es constantemente idéntico a sí mismo. El segundo es objeto de la opinión unida a la sensación irracional, ya que nace y muere, pero no existe jamás realmente" (Platón, 1969, 26e/28c p.1133). Además, la "aprehensión" de la cual habla, según su definición de saber científico (episteme) dada en el Teeteto, como "juicio verdadero acompañado de razón" (Platón, 201e/203b p.935), es precisamente ideal (eidos), representada en la verdad matemática al tomar como arquetipo de "razón" el teorema de Pitágoras, esto es, la relación precisa entre los conceptos aritméticos y geométricos en correspondencia con su idealización de la physis.

Lo anterior es el argumento por el cual, históricamente, en el dominio de las ciencias, se ha asociado el devenir con lo irracional. Indudablemente el devenir expone problemas que demandan de otras matemáticas y lo cierto es que no se contaba con las estructuras matemáticas que pudieran explicar y demostrar tal complejidad en la naturaleza hasta la reciente creación de la termodinámica no-lineal, la teoría del caos, la topología, la teoría de las catástrofes, la geometría fractal, entre otras. En efecto, son matemáticas de cambios cualitativos impredecibles, de patrones de comportamiento de poblaciones, en síntesis, de transformaciones morfológicas de sistemas que evolucionan en función de múltiples interacciones, entre sí y con el entorno, y, por tanto, en múltiples dimensiones e intensidades temporales ya que cada interacción expresa una diferencia esencialmente temporal, un «encuentro». Tal es el caso, por ejemplo, de los sistemas cuyo comportamiento expresa complejidad creciente, como la evolución de vida en la tierra (la biosfera), los cuales explicaremos enseguida a través de la conceptualización de las estructuras disipativas.

\footnotetext{
Ver, por ejemplo, Margulis, L. \& Sagan, D. (2001). Igualmente, Leakey, R. \& Lewin, R. (1997).

En tal sentido es recomendable Sotolongo, P. L. (2006). Teoría social y vida cotidiana: La sociedad como sistema dinámico complejo. La Habana: Publicaciones Acuario, Centro Félix Varela.
}

Rev. Invest. Univ. Quindío (20): 78 - 88. Armenia - Colombia 
Comparativamente, mientras que la comprensión tradicional del Ser (inmutable) se relaciona con el tipo de saber que fija la identidad en la dinámica lineal y (o) periódica, es decir, con el mero movimiento de los entes expresable en trayectorias; la comprensión del devenir remonta lo anterior (no lo rechaza pero sí hace impotente su simplificación) al vincularse con el cambio y la transformación morfológica de los componentes del mundo en incesante actualización de su diferenciación. "Llegamos así a dos descripciones sustancialmente diferentes: la dinámica, aplicable al mundo de las masas en movimiento, y la termodinámica, base de la ciencia de lo complejo: Pero ante esta dualidad se plantea infaliblemente esta pregunta: ¿cómo articular esas descripciones?" (Prigogine \& Stengers, 1997, p. 161). Lo que está en juego es la afirmación del pensamiento paradojal, es decir, aquel en y con el cual se supera la fosilización de la lógica del tercero excluso ${ }^{10}$, en -por- la plurivalencia o multiplicidad expresada en la conjunción y la afirmación de futuros posibles (como, por ejemplo, con la invención de valor imaginario en matemáticas): devenir-entre-las-diferencias. En tal sentido lo concibe Deleuze según la exégesis de François Zourabichvili:

Reste à comprendre comment la différence peut réunir, et le multiple être dit une multiplicité. C'est que la différence ainsi définie a un corrélat : la répétition. La différence ne cesse de revenir dans chacune de ses différenciations, dans chacune des différences. Le paradoxe est immédiatement visible : la différence se répète en se différencient, et pourtant ne se répète jamais à l'identique (une telle idée serait évidemment absurde dans le champ de la représentation, où la répétition se confond avec la reproduction du même; elle n'en est que plus délicate à penser). La différentiation de la différence a pour corrélat une répétition qui diverge ou qui sonne faux, et Différence et répétition est la logique de la multiplicité intensive comme concept du temps. Chaque fois la différencedimension revient, mais elle revient en différant, donc à un autre niveau, sur un autre plan, dans une autre dimension. L'interprétation deleuzienne de l'eternel retour chez Nietzsche repose sur cette corrélation de la différence et de la répétition (d'où un rapport très particulier du passé et du futur, de la mémoire et de la croyance $)^{11}$. (1996, p.84)

Es notable que la termodinámica esté cambiando nuestra concepción del mundo, especialmente en lo relativo al papel constructivo del tiempo relacionado con los fenómenos irreversibles del cual Prigogine ha sido su más destacado investigador. El resultado más significativo, como es ampliamente conocido, lo obtuvo a través del estudio físicoquímico de sistemas abiertos alejados del equilibrio a partir de los cuales creó el concepto de estructura disipativa. Pues bien, dicho concepto es clave para abordar, entre otras cuestiones, lo inherente a comportamientos complejos presentes en diversos sistemas, los cuales han sido y son estudiados en las ciencias de la vida, ciencias de la salud, ciencias económicas, ciencias de la tierra, ciencias sociales humanas, entre otras. De tal modo que, la naturaleza compleja de los problemas que surgen al trabajar con sistemas abiertos alejados del equilibrio, hace indispensable este concepto en el pensamiento contemporáneo. Justamente, lo característico en estos comportamientos complejos es que lo propio del equilibrio, unicidad y estabilidad, que llevan a la "destrucción de estructuras», como dice Nicolis (2000), quedan comprometidas, y es allí donde el concepto de estructura disipativa es correlativo.

Precisamente, en problemas socio-económicos, sociobiológicos, ecológicos, en general, en todos aquellos que expresan cuestiones básicas de la vida social humana, más aún, de la vida en general, es propio encontrar diversidad e inestabilidad. Es lo propio de los sistemas abiertos alejados del equilibrio, aquellos que, como se ha indicado (figuras 3 y 4), intercambian materia, energía e información con su entorno. Que, además, en función de las fluctuaciones surgidas del flujo de interacciones en la diversidad o en el encuentro de diferencias que ocasionan inestabilidad o crisis al sistema, se posibilita su autoorganización (o adaptación) en la creación de nuevo orden, esto es, la emergencia de nuevas estructuras sensibles a acontecimientos repentinos que nuevamente la desestabilizan. Éstas son las llamadas "estructuras disipativas» y los puntos de inestabilidad que les dan origen son los que Prigogine denomina «puntos de bifurcación». Quiere decir, entonces, que, en y con el tiempo, la emergencia de estructuras o nuevos ordenamientos constituyen las bifurcaciones del sistema, sus huellas estructurales, o sea, su narrativa, su historia, su sentido evolutivo.

Las bifurcaciones están en el origen de todo viviente: organismo u organización. Hubo un momento en la evolución de la vida en la tierra, hace aproximadamente 530 millones de años, donde se presenta la sorprendente eclosión de la biodiversidad, período que los paleontólogos denominan como «explosión cámbrica». Allí, "en unos cuantos millones de años, en un brote de innovación evolutiva, se inventaron los principales planes estructurales,

\footnotetext{
${ }^{10}$ Llama la atención, en efecto, que en la actualidad, específicamente a partir de la segunda mitad del siglo XX, el campo de trabajo de los lógicos se caracteriza cada vez más por los desarrollos en lo que se ha denominado lógicas no-clásicas o lógica filosófica. En rigor, consiste en la afirmación del pluralismo lógico y, con ello, de la diversidad de formas de pensar y decir la relación de consecuencia lógica, entre otros asuntos. Algunas de estas lógicas son: lógica modal, lógicas paraconsistentes, lógica de la relevancia, lógicas polivalentes, lógica difusa, lógica temporal, lógica cuántica, lógica de la reflexividad, entre otras. Véase, por ejemplo, Peña, L. (1993). Introducción a las lógicas no clásicas. Universidad Nacional Autónoma de México. Palau, G. (2002). Introducción filosófica a las lógicas no clásicas. Barcelona: Gedisa. Maldonado, C.E. (2005). ¿Por qué hay múltiples lógicas?, En: Zero, No.15. Bogotá: Universidad Externado de Colombia, pp. 213-217.

${ }^{11}$ Queda por comprender cómo la diferencia puede reunir, y lo múltiple ser llamado una multiplicidad. Porque la diferencia así definida tiene un correlato: la repetición. La diferencia no cesa de volver en cada una de sus diferenciaciones, en cada una de las diferencias. La paradoja es inmediatamente visible: la diferencia se repite diferenciándose, y sin embargo no se repite jamás en lo idéntico (una idea tal sería evidentemente absurda en el campo de la representación, donde la repetición se confunde con la reproducción de lo mismo; lo que la hace más complicado de pensar). La diferenciación de la diferencia tiene por correlato una repetición que diverge o que suena falsa, y Diferencia y repetición es la lógica de la multiplicidad intensiva como concepto del tiempo. Cada vez la diferencia-dimensión vuelve, pero vuelve difiriendo, por lo tanto en otro nivel, sobre otro plano, en otra dimensión. La interpretación deleuziana del eterno retorno en Nietzsche descansa sobre esta correlación de la diferencia y la repetición (de donde emerge una relación muy particular del pasado y el futuro, de la memoria y la creencia). Nota: esta traducción, no oficial, es del autor de este artículo.
} 
o tipos, que representan la vida actual" (Leakey \& Lewin, 1997, p. 26). Y en este flujo azaroso de bifurcaciones que ha sido la historia de la biosfera, entre períodos de cambios graduales y catastróficos cuyas únicas triunfantes han sido la adaptación y la innovación, emergió recientemente, hace cerca de 100.000 años, precisamente como una bifurcación más, el sistema nervioso y cerebro del Homo sapiens y, con éste, su potencial de racionalidad y de creación de culturas. De este modo, análogo a como del caos surge nuevo orden, de lo "irracional", del azar, surgió lo racional. Son las paradojas del devenir-entre-las-diferencias. Finalmente, la creatividad humana es una expresión de lo que siempre ha hecho la naturaleza.

Esta reciente concepción del mundo a la que el pensamiento contemporáneo nos ha permitido acceder en torno al tiempo, tiene un significativo valor para nuestra cultura. En efecto, como lo plantea Prigogine y Stengers en "La nueva alianza", superar dos tendencias que históricamente han estado contrapuestas: la cultura científica mecanicista y la cultura humanista. La primera asociada a lo racional en función de la certeza; la segunda asociada al cambio y, como hemos dicho, a lo se asumía como irracional. No obstante, en un mundo en autoconstrucción, donde el futuro está haciéndose en la combinatoria de condicionamientos e historia, la reversibilidad y la simplicidad del dominio explicativo de la ciencia clásica se muestran como casos particulares.

Ninguna teoría científica debería bastar, como tal ciencia, para justificar una reducción del tiempo semejante a la que realiza la dinámica clásica. Negar el tiempo, esto es reducirlo al desarrollo determinista de una ley reversible, es renunciar a la posibilidad de definir una naturaleza capaz de producir seres vivos y, en particular, el hombre; es condenarse a escoger entre una filosofía anticientífica y una ciencia alienante (Prigogine \& Stengers, 1997, p. 136).

De hecho, todo en lo humano (en sus múltiples dimensiones) como componente de la biosfera, son estructuras históricas entretejidas: la cognición, la familia, la amistad, las organizaciones, los países, la política, la cultura, etc. Por tanto, lo humano deviene como una expresión fractal de la evolución del mundo. Tanto así que nuestra composición bioquímica es semejante a la del universo. "Nuestros organismos aún son muy ricos en hidrógeno (tenemos mayor cantidad de átomos de hidrógeno que de cualquier otro elemento), principalmente en forma de agua. Nuestros cuerpos de hidrógeno son el reflejo de un universo de hidrógeno" (Margulis \& Sagan, 2001, p. 58). En definitiva, actualmente no es sostenible la contraposición entre lo natural y lo humano en el dominio de las ciencias; un planteamiento en tal sentido caería en la negación del mundo, de su evolución temporal, pasando primero por la negación de quién lo formula.

Pues bien, como se ha ido explicitando, el pensamiento contemporáneo es fecundo en sus alcances en tres planos interdependientes: en la comprensión de sistemas de comportamiento de complejidad creciente; en la explicación-demostración de la no-linealidad de dicho comportamiento; y en el de decisión-acción en un mundo vivo donde todos sus componentes -humanos y nohumanos-fluyen en una red de rizos como en la «trama de la vida» expuesta por Fritjof Capra. Para esclarecer aún más lo dicho hasta aquí, y como recurso ilustrativo, podemos observar la siguiente tabla.

Los orígenes de cada dominio explicativo, para no dar cabida

\begin{tabular}{|c|c|c|}
\hline $\begin{array}{c}\text { Dominio explicativo clásico } \\
\text { En función de trayectorias } \\
\text { Orígenes: Pitágoras, Parménides, Platón y Aristóteles }\end{array}$ & $\ldots$ & $\begin{array}{c}\text { Dominio explicativo contemporáneo } \\
\text { En función de t ransformaciones } \\
\text { Orígenes: Heráclito, Demócrito, Epicuro y Lucrecio ... }\end{array}$ \\
\hline Unidad & & Multiplicidad y unidad \\
\hline Simplicidad & & Complejidad y simplicidad \\
\hline Orden & & Caos y orden \\
\hline Determinismo & & Azar y determinismo \\
\hline Permanencia & & Cambio (diferencia) y permanencia (repetición) \\
\hline Causalidad (linealidad y simetría) & & Emergencia y causalidad \\
\hline Cuantificación (número) & & Cualificación (c oncepto) y cuantificación \\
\hline Predicción & & Previsión (de prever) y predicción \\
\hline Probabilidad & & Posibilidad y probabilidad \\
\hline Experimentación & & Simulación y experimentación \\
\hline Ética & & Bios y ethos (Bioética) \\
\hline
\end{tabular}

a equívocos, plantean una distinción ontológica en sus estructuras $y$, desde luego, en sus correspondientes epistemologías y lógicas. De este modo, los puntos sucesivos en el recuadro izquierdo indican el desarrollo normal del pensamiento en occidente, pasando por la metafísica o la teología hasta llegar a la recta razón: canon del pensar desde el siglo XVII hasta bien entrado el XX donde se equipara lo racional y la ciencia, esto es, donde la ciencia, convertida en núcleo de poder, hace "sociedad", "cuerpo" y "mundo"12 de espaldas a éste. En efecto, el dominio explicativo del

\footnotetext{
${ }^{12}$ Aunque son evidentes ciertos éxitos de la ciencia moderna, igualmente lo son algunos resultados atroces de su aplicación. En tal sentido es que la ciencia y la tecnología comienzan a ser objeto de reflexión ética, social y ecológica en los estudio de «ciencia, tecnología y sociedad», entre otros. Véase, por ejemplo, Giddens, A., Bauman, Z., Luhmann, N. \& Beck U. (1996). Las consecuencias perversas de la modernidad. Modernidad, contingencia y riesgo. Barcelona: Anthropos.
}

Rev. Invest. Univ. Quindío (20): 78 - 88. Armenia - Colombia 
pensamiento clásico, atrapado en los principios lógicos de identidad, no-contradicción y tercero excluso, aboga por la disyunción, la exclusión de lo singular, la expulsión o aniquilación de la diferencia, la definición y el encierro. Es por ello que la razón moderna, afincada también en la técnica, devino en horror para la vida.

Por otro lado, con otro alcance conceptual (ontológico y epistemológico) se evidencia que los componentes del dominio explicativo del pensamiento contemporáneo se caracterizan por la conjunción, las interacciones entre diferentes escalas de la diversidad y el devenir como condición del proceso de creación de diferencia, esto es, invención de futuros posibles. Así pues, pensar en y con el tiempo, en cualquier dominio, es una decisión que implica ineludiblemente un compromiso ontológico que lo afirme, un habitar que, en semejanza con la vida, crea porvenir; en definitiva, remontar lo que Deleuze denomina la «imagen dogmática del pensamiento». ${ }^{13}$

Así mismo, el dominio contemporáneo, no acaece como antítesis del pensamiento clásico, lo cual significaría continuar atrapado en la exclusión y lo idéntico al denotar dicotomía. Ni como síntesis puesto que, igualmente, la conciliación de contrarios, sugiere contradicción. Ni lo uno, ni lo otro. Este dominio acontece plural, esto es, con en lugar de contra. Efectivamente, las fluctuaciones en las estructuras disipativas se dan entre la diferencia, al interior del sistema y de éste con el entorno. Esta sencilla comprensión ha permitido el diálogo de saberes que hace posible la investigación inter y transdisciplinaria y el trabajo intercultural para hacer frente al enorme desafío de la actualidad, a saber, el destino de lo humano jugado en el de la biodiversidad del planeta y en el tipo de manejo que demos a los riesgos globales.

\section{CONCLUSIÓN.}

El concepto del tiempo como devenir en el dominio explicativo de las ciencias constituye una verdadera novedad en el pensamiento, ¿realmente hemos tenido "revoluciones científicas» antes del siglo XX o se ha tratado de reformas parciales que han alcanzado un punto crítico en la ciencia contemporánea? El estudio de los sistemas abiertos alejados del equilibrio nos ha permitido comprender y explicar matemáticamente el fluir del mundo en función de sus diferencias, donde lo humano cohabita como otra diferencia, continuamente actualizándose. Esto era impensable hasta hace muy poco en las ciencias dominadas por el ideal clásico que apela a la razón y al método como principios universales en orientación hacia el presupuesto de objetividad.

De ahí que, precisamente porque es cierto que nuestra época se caracteriza por tener problemas en cuya evolución se integran múltiples grupos e individuos de diversas especies y en diferentes escalas, es decir, problemas que se ubican en los más altos niveles de complejidad, son innegable las apuestas que el pensamiento contemporáneo viene haciendo para hacer frente a ese tipo de dificultades que nos son propias. Remontar el tiempo y el lenguaje de la descriptiva clásica (de trayectorias) es una necesidad que el mundo expresa cada vez con mayor urgencia: ser comprendido con renovadas herramientas conceptuales y tecnológicas y, desde luego, ser habitado sosteniblemente en lo socioeconómico pero, principalmente, en lo ecológico.

El dominio explicativo contemporáneo realmente entabla un diálogo con la naturaleza, reconociendo la historia de la vida humana en la historia de la biosfera, esto es, reconociéndose en lo que percibe. De este modo es que la cultura científica y la cultura humanista ya no están contrapuestas ( «La nueva alianza») pues tienen el problema del tiempo (un mundo de estructuras disipativas en interacción, narrándose) como elemento común. Esto caracteriza una época de transformación asociada principalmente a la indeterminación y la creatividad, es decir, al devenir.

\section{BIBLIOGRAFÍA}

Deleuze, G. (2002). Nietzsche y la filosofía. Barcelona: Anagrama.

Grondin, J. (2006). Introducción a la metafísica. Barcelona: Herder.

Hume, D. (1984). Del conocimiento. Madrid: Sarpe.

Kant, I. (1998). Crítica de la razón pura. Madrid: Alfaguara.

Khun, T. S. (1995). ¿Qué son las revoluciones científicas? Barcelona: Altaya.

Khun, T. S. (2004). La estructura de las revoluciones científicas. México, D. F.: FCE

Leakey, R. \& Lewin, R. (1997). La sexta extinción, el futuro de la vida y de la humanidad. Barcelona: Tusquets.

Leibniz, G. W. (1983). Monadología. Barcelona: Orbis.

\footnotetext{
${ }^{13}$ «La imagen dogmatica del pensamiento aparece en tres tesis esenciales: 1o. Se nos dice que el pensador en tanto que pensador quiere y ama la verdad (veracidad del pensador); que el pensamiento como pensamiento posee o contiene formalmente la verdad (connaturalidad de la idea, a priori de los conceptos); que el pensar es el ejercicio natural de una facultad, que basta pues pensar «verdaderamente» para pensar con verdad (recta naturaleza del pensamiento, buen sentido, compartido universalmente); 2o. Se nos dice también que hemos sido desviados de la verdad, pero por fuerzas extrañas al pensamiento (cuerpos, pasiones, intereses sensibles). Porque no solo somos seres pensantes, sino que caemos en el error, tomamos lo falso por lo verdadero. El error: este sería el único efecto, en el pensamiento como tal, de las fuerzas exteriores que se oponen al pensamiento. 3o. Finalmente, se nos dice que basta un método para pensar bien, para pensar verdaderamente. El método es un artificio, pero gracias al cual encontramos la naturaleza del pensamiento, nos adherimos a esta naturaleza y conjuramos el efecto de las fuerzas extrañas que la alteran y nos distraen. Gracias al método conjuramos el error. Poco importa el lugar y la hora si aplicamos el método: este nos introduce en el dominio de lo que vale en todo tiempo y lugar» (Deleuze, 2002, p. 146). Véase también Deleuze, G. (2002). Diferencia y repetición. Buenos Aires: Amorrortu ediciones. pp. 201 - 254.
} 
Maldonado, C.E. (2005). ¿Por qué hay múltiples lógicas?, En: Zero, No.15. Bogotá: Universidad Externado de Colombia. Margulis, L. y Sagan, D. (2001). Microcosmos, cuatro mil millones de años de evolución desde nuestros ancestros microbianos. Barcelona: Tusquets.

Newton, I. (1994). Principios Matemáticos. Barcelona: Altaya.

Nicolis, G. y Prigogine, I. (1997). La estructura de lo complejo. El camino hacia una nueva comprensión de las ciencias. Madrid: Alianza.

Nisolis, G. (2000). Estructuras disipativas, bifurcaciones y fluctuaciones: hacia una dinámica de los sistemas complejos. En: Tiempo y devenir. Barcelona: Gedisa.

Palau, G. (2002). Introducción filosófica a las lógicas no clásicas. Barcelona: Gedisa.

Peña, L. (1993). Introducción a las lógicas no clásicas. Universidad Nacional Autónoma de México.

Platón, (1969). Obras completas. Madrid: Aguilar.

Prigogine I. y Stengers, I. (1994). Entre el tiempo y la eternidad. Madrid: Alianza.

Prigogine I. y Stengers, I. (1997). La nueva alianza. Metamorfosis de la ciencia. Madrid: Alianza. Prigogine, I. (1997). ¿ ¿Tan sólo una ilusión? Una exploración del caos al orden. Barcelona: Tusquets.

Zourabichvili, F. (1996). Deleuze une philosophie de l'événement. Paris: Presses universitaires de France.

Spire, A. (2001). El pensamiento de Prigogine. La belleza del caos. Barcelona: Andrés bello.

Serres, M. (1994). El nacimiento de la física en el texto de Lucrecio. Caudales y turbulencias. Madrid: Cátedra.

Serres, M. (ed.) (1998). Historia de las Ciencias. Madrid: Cátedra. 